\title{
Interaction with Virtual Agents - Comparison of the Participants' Experience Between an IVR and a Semi-IVR System
}

\author{
Marios Kyriakou* \\ Department of Computer Science \\ University of Cyprus
}

\author{
Xueni $\mathrm{Pan}^{\dagger}$ \\ Institute of Cognitive Neuroscience \\ University College London
}

\author{
Yiorgos Chrysanthou ${ }^{+}$ \\ Department of Computer Science \\ University of Cyprus
}

\begin{abstract}
In this paper we compare participants' behavior and experience when navigating through a virtual environment populated with virtual agents in an IVR (Immersive Virtual Reality) system and a semi-IVR system. We measured the impact of collision and basic interaction between participants and virtual agents in both systems. Our findings show that it is more important for our semiIVR systems to facilitate collision avoidance between the user and the virtual agents accompanied with basic interaction between them. This can increase the sense of presence and make the virtual agents and the environment appear more realistic and lifelike.
\end{abstract}

\section{INTRODUCTION}

Virtual agents are an important component for many VR applications in both IVR and semi-IVR systems. Several studies have shown that participants react and behave towards virtual agents in a highly realistic manner [1]. Even if participants are aware that virtual agents are computer characters, they automatically attribute mental states to them [2].

Many experimental studies have been conducted in the area of understanding the interaction between virtual agents and human participants. Still, for IVR and semi-IVR systems there is not sufficient background work on how different levels of interaction between the participant and the virtual crowd affect the participant's behavior, perception of realism and sense of presence and if there are any differences in these two types of systems. In this study, we concentrate on the comparison of the behaviors of the participants between these two systems focusing on two main virtual agents' behavior characteristics, collision avoidance and basic interaction between the virtual crowd and the participant.

\section{RELATED WORK}

The distance that participants tend to maintain with a virtual agent appears to be an important issue, since it was found that a greater distance is maintained with more realistic agents [3] and negative reactions can be caused due to violations of interpersonal space [4]. These motivated us to study further how collisions between virtual agents and the participants affect the participants' behavior. Interaction is another important issue of how participants are influenced by virtual agents in VR systems. Garau et al. [5] found that if there is no interaction between the participant and the virtual agents, then any sense of presence is eliminated.

We have recently conducted an experimental study that showed that facilitating collision avoidance between the user and the virtual agents does not guarantee that the VR system will be more

\footnotetext{
*mkyriakou@gmail.com

${ }^{\dagger}$ s.pan@cs.ucl.ac.uk

+yiorgos@cs.ucy.ac.cy
}

IEEE Virtual Reality Conference 2015

23 - 27 March, Arles, France

978-1-4799-1727-3/15/\$31.00 @2015 IEEE plausible or easy to use [6]. We found that collision avoidance should be accompanied with basic interaction between the user and the virtual agents in order to increase the plausibility, the feeling of comfort and the sense of presence of the participant. Using those results, we are studying in this research to find any differences between the participants' behavior, perception of realism, and their sense of presence, in the IVR and in the semiIVR system.

\section{Method}

We have conducted two experimental studies with 50 participants in total: 30 of them participated in a semi-IVR system experiments and 20 in an IVR system. Each volunteer participated in three different scenarios in a random order. The design of the experiment was repeated-measures (within-subjects). After each scenario, the participants were asked to fill in a web-based questionnaire on a Likert scale ranging from 1 to 5 (1=Minimum, $5=$ Maximum).

\subsection{The systems}

Immersive VR System: A Cave-like projection based system where participants' heads were tracked with an Intersense IS 900 tracker, and the navigation was facilitated through a wand.

Semi - Immersive VR System: A custom-built semi-IVR system, using a large screen front-projected wall, driven by a workstation. Using a Kinect for motion sensing and human body tracking, the participants were able to navigate.

\subsection{Scenarios}

We designed a 3D virtual environment representing an open-space mall with a significant number (33) of animated virtual characters.

The participants were given the task to locate a virtual child and follow her wherever she went. This was their primary goal and was clearly stated to them. The virtual child was programmed to follow a trajectory, where she came across other virtual characters, and avoiding collisions with them.

Three different scenarios were created with different level of interaction between the virtual agents and the user:

Scenario 1 (S1): the virtual agents ignore the participant (the virtual characters do not avoid any collision with the participant, and have no other interaction with him/her).

Scenario 2 (S2): the agents avoid collisions with the participant but have no other interaction.

Scenario 3 (S3): the agents interact with the participant using some basic socialization (talking to him, looking at him, waving etc.) as well as applying collision avoidance with the participant.

\subsection{Results}

For the statistical analysis of the answers we used the Friedman test for IVR $(n=20)$ and for semi-IVR $(n=30)$ to find the differences between the three scenarios. Then we used the Wilcoxon signed-rank to evaluate the significance of any increase/decrease from a scenario to a scenario for both systems (see Figure 1). 
Aware_self concerned the crowd's awareness of the participant. The perceived virtual crowd's awareness of themselves was significantly different between the three scenarios in both IVR and semi-IVR. In the semi-IVR system this feeling was stated as stronger in S3. Aware_others concerns how the virtual characters were aware of each other, which was programmed to be at the same level across the three scenarios; however the participants, falsely believed that it had been raised from S1 to S2. In the semiIVR system this was even stronger in S3. For both systems users thought that it was easier for them to use the system in S2, where the most difficult was stated to be in S3 (Easiness). The evaluation of the question concerning the sense of presence (Presence) delivered responses as expected in both systems. The lower level of presence was stated to be in S1 and the higher was in S3. The differences between the stated levels were higher in the semi-IVR system. The question inquiring about the participants' feeling of comfort in the system (Comfort), showed the same responses as for Easiness. Users stated the lower level of comfort in S2 and the higher in S3. The question Realism_Child addressed the perceived realism of the child. This was stated to be higher in $\mathrm{S} 3$ and lower in S1. The realism of the crowd the rest of the virtual characters- was stated as significantly different in all scenarios (Realism_Crowd) for both systems. The virtual environment was exactly the same in all three scenarios. Nevertheless, the answers of the users of the semi-IVR system showed statistically significant differences in the question Realism_Env. The realism of the environment in $\mathrm{S} 2$ was perceived as better than in $\mathrm{S} 1$, and in $\mathrm{S} 3$ better than $\mathrm{S} 2$.

\section{Conclusion}

In this study we compared the participants' answers when they interact with virtual agents in both IVR and semi-IVR systems and found significant outcomes. Firstly, in most questions, participants gave similar answers for both systems: they found it less comfortable and more difficult to navigate in the VR with collision avoidance than without. Secondly, extending the relationship between the user and the virtual agents with some basic level of interaction between them made the user's experience even more positive. The evaluation of all examined factors by the user was considerably better when there was a basic level of interaction with the virtual crowd. The behavior of the crowd was perceived as more realistic and the user reported a stronger sense of presence. This finding is even more important in the semi-IVR system since in some questions (awareness of the others, awareness of himself, realism of the environment, sense of presence) the differences were greater. This can be explained if we consider that in a semi-IVR system the user is not fully immersed and the factors that contribute to the plausibility of the system and the sense of presence are more "weak" than in an IVR system. Thus, the introduction of collision avoidance accompanied with basic interaction between the user and the virtual agents is perceived by the users in some aspects as more important, in terms of realism and sense of presence in our semiIVR system.

Further work is necessary to study more factors that affect the user experience when they participate in populated IVE with virtual agents in both IVR and semi-IVR systems. For example, it would be useful to examine the plausibility of different agents' group formations and sizes and if these affect differently the users in an IVR from the users in a semi-IVR.

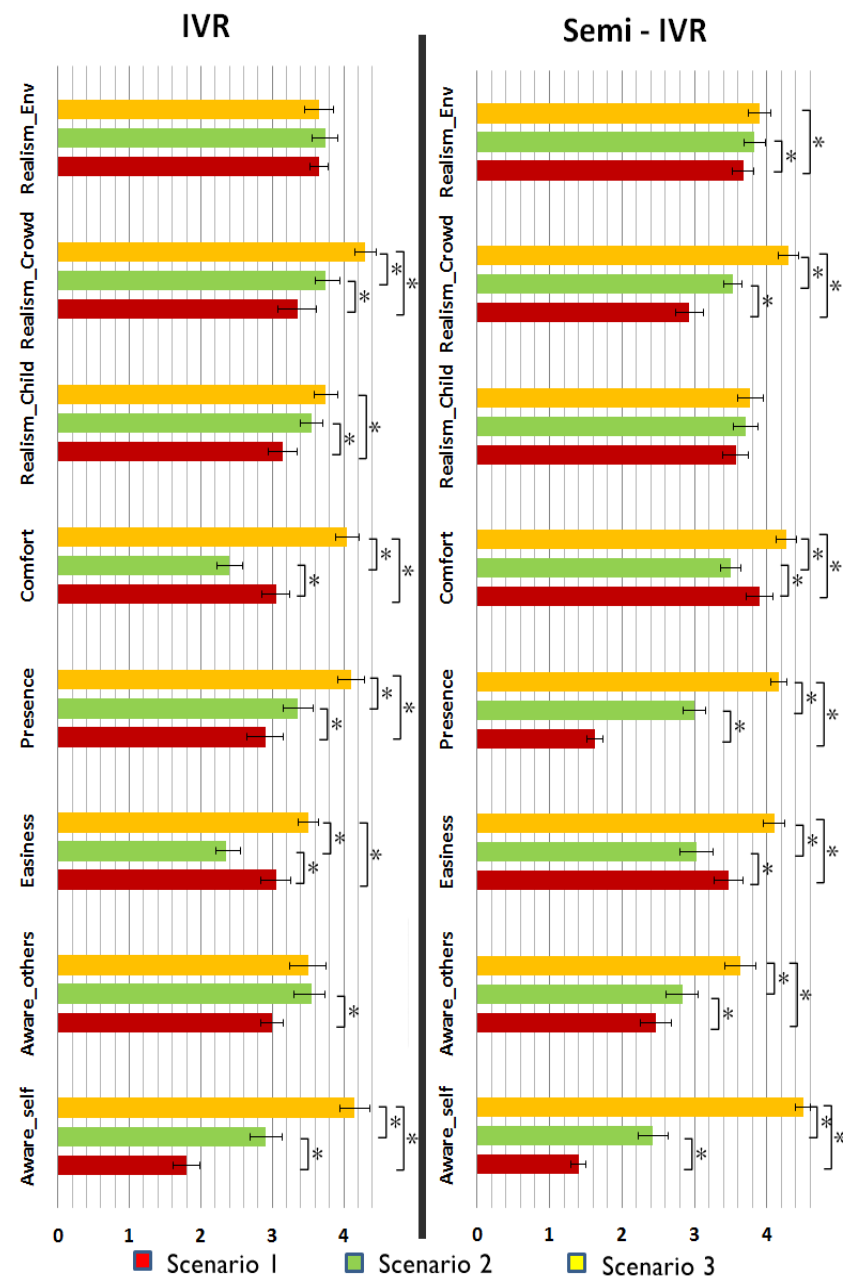

Figure 1: Results of the statistical analysis of the users' answers on both systems (IVR and semi-IVR). Error bars present standard error of means. ${ }^{*}=p<0.05$.

\section{RefERENCES}

[1] D. Pertaub, M. Slater, and C. Barker, "An experiment on public speaking anxiety in response to three different types of virtual audience," Presence Teleoperators Virtual Environ., vol. 11, no. 1 , pp. 68-78, 2002.

[2] D. Freeman, M. Slater, P. E. Bebbington, P. A. Garety, E. Kuipers, D. Fowler, A. Met, C. M. Read, J. Jordan, and V. Vinayagamoorthy, "Can virtual reality be used to investigate persecutory ideation?," J. Nerv. Ment. Dis., vol. 191, no. 8, pp. 509-514, 2003.

[3] J. N. Bailenson, J. Blascovich, A. C. Beall, and J. M. Loomis, "Equilibrium Theory Revisited: Mutual Gaze and Personal Space in Virtual Environments," Presence: Teleoperators and Virtual Environments, vol. 10, no. 6. pp. 583-598, 2001.

[4] L. M. Wilcox, R. S. Allison, S. Elfassy, and C. Grelik, "Personal space in virtual reality," ACM Transactions on Applied Percepion., vol. 3, no. 4, pp. 412-428, 2006.

[5] M. Garau, D. Friedman, H. Ritter Widenfeld, A. Antley, A. Brogni, and M. Slater, "Temporal and Spatial Variations in Presence: Qualitative Analysis of Interviews from an Experiment on Breaks in Presence," Presence Teleoperators Virtual Environ., vol. 17, no. 3, pp. 293-309, 2008.

[6] M. Kyriakou, X. Pan, and Y. Chrysanthou, "Interaction with Virtual Crowd in Immersive and semi- Immersive Virtual Reality systems.," Submitted in Transactions on Applied Perception, 2014. 